\title{
Determination of Pinaverium Bromide in pharmaceutical dosage forms by a validated Stability-Indicating LC Method
}

\author{
Maximiliano da Silva Sangoi*, Anna Karolina Mouzer da Silva Machado, Lorena Coimbra Florindo, Brenda Rocha \\ Gonçalves, Vítor Todeschini \\ Laboratory of Pharmaceutical Analysis, Faculty of Pharmacy, Federal University of Rio de Janeiro, 27930-560 Macaé- \\ RJ, Brazil \\ * Corresponding author: e-mail: maxsilvasangoi@yahoo.com.br
}

\begin{abstract}
A reversed-phase liquid chromatography (LC) method was validated for the determination of pinaverium bromide (PB) in pharmaceutical dosage forms. The LC method was carried out on a reversed-phase monolithic $\mathrm{C} 18$ column (100 x 4.6 $\mathrm{mm}$ i.d.), maintained at $45^{\circ} \mathrm{C}$. The mobile phase consisted of acetonitrile and a solution of triethylamine $0.3 \% \mathrm{pH} 5.0$ $(50: 50 ; \mathrm{v} / \mathrm{v})$, run at a flow rate of $2.0 \mathrm{~mL} / \mathrm{min}$, with photodiode array detector set at $213 \mathrm{~nm}$. The chromatographic separation was obtained with PB retention time of $3.4 \mathrm{~min}$, and it is linear in the range of $5-100 \mu \mathrm{g} / \mathrm{mL}\left(R^{2}=0.9991\right)$. The limits of detection and quantitation are 1.41 and $4.70 \mu \mathrm{g} / \mathrm{mL}$, respectively. The specificity and stability-indicating capability of the method were proven through degradation studies, which also showed that there is no interference of the formulation excipients, showing that peak is free from any co-eluting peak. The method showed adequate precision, with a relative standard deviation values lower than $1.38 \%$. Excellent values of accuracy were obtained, with a mean value of $100.68 \%$. Experimental design was used during validation to calculate and prove method robustness. The proposed LC method is applied for the analysis of the PB pharmaceutical dosage forms, contributing to improve the quality control and to assure the therapeutic efficacy.
\end{abstract}

Keywords: pinaverium bromide; experimental design; liquid chromatography; stability-indicating method; validation.

\section{Introduction}

Irritable bowel syndrome (IBS) is the most commonly diagnosed functional gastrointestinal disorder characterized by chronic abdominal pain and changed bowel habits in the absence of an overtly identifiable cause (1). The prevalence of IBS in Europe and North America is estimated to be $10-15 \%$ and up to $33 \%$ in the worldwide population (2). IBS has gained significant interest in healthcare due to its high occurrence, complex pathophysiology, difficult diagnosis due to a wide range of non-specific symptoms, and varied and challenging treatment options (3).

Antispasmodics improve IBS symptoms by directly relaxing the colonic smooth muscle cells or antagonizing the excitatory neuromuscular neurotransmission. Antispasmodics remain one of the most commonly prescribed groups of medications for the treatment of IBS (4). Pinaverium bromide (PB; Figure 1), an antispasmodic, is a commonly drug administered worldwide (5).

Liquid chromatography (LC) is a versatile separation technique with wide range of applications, usually the analytical method of choice for pharmaceutical quality control and impurity profile $(6,7)$. However, there are still ample opportunities for improvements based on specificity and sensitivity, enhancing robustness or improving cost effectiveness, such as chemometry and column technology.

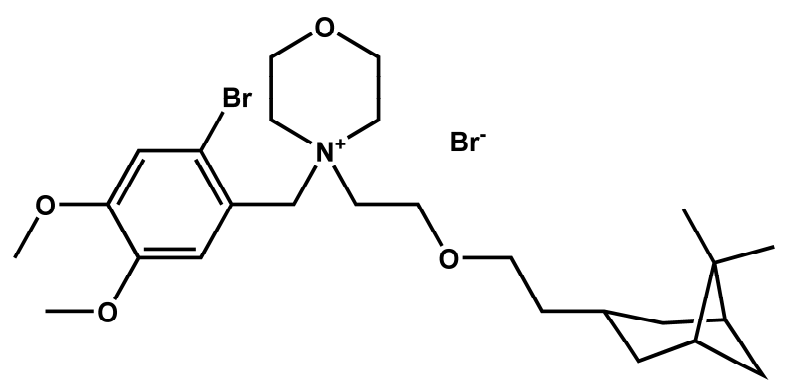

Figure 1. Chemical structure of bromide pinaverium.

Chemometric approaches have been increasingly viewed as precious complements to chromatographic practices, since a large number of variables can be simultaneously controlled to achieve the desired separations. Moreover, applications of suitable statistical analysis may efficiently identify and optimize the significant factors to accomplish competent results through limited experimental trials with lower reagent consumption and less laboratory work $(8,9)$.

There is a continuous development of new column materials, which may be used in the separation of impurities given that this will offer an advantage over legacy materials (10). An interesting alternative to particulate-based LC columns are monolithic columns. 
Silica monoliths are ideal for small molecule applications with high efficiencies, higher flow rates and lower operating pressures, successfully applied to impurity profiling and quality control (11-13).

For the analysis of PB in tablets, two spectrophotometric methods have been developed by our research group (14). However there is no publication concerning LC method in the current literature. Therefore, the aim of the present article was to develop a selective, simple, rapid and validated stability-indicating LC method on a monolithic reversed-phase column to perform forced decomposition studies, contributing to improve the quality control and ensuring therapeutic efficacy.

\section{Experimental}

\section{Chemicals and reagents}

The PB reference substance (purity 100.3\%) was acquired from the Pharmacopeia Comércio e Importação Ltda (Barueri, SP, Brazil). Dicetel ${ }^{\circledR}$ tablets (Abbott Laboratories Ltda, SP, Brazil) containing 50 $\mathrm{mg}$ and $100 \mathrm{mg}$ of PB were obtained from commercial source and used within their shelf life period. The excipients contained in the dosage form were all of pharmaceutical grades and acquired from different suppliers for placebo preparation. LC-grade triethylamine, phosphoric acid $85 \%$ and acetonitrile were obtained from Tedia Company Inc (Fairfield, OH, USA). Ultrapure water (Milli-Q ${ }^{\circledR}$ Direct Water Purification System, Merck Millipore Corporation, Darmstadt, Germany) was used for all the analyses.

\section{Sample preparations}

The stock solution of the drug was prepared by accurately weighing $20 \mathrm{mg}$ of PB reference substance and diluting to volume with acetonitrile to obtain the concentration of $1.0 \mathrm{mg} / \mathrm{mL}$. The stock solution was stored at $2-8{ }^{\circ} \mathrm{C}$, protected from light and daily diluted to an appropriate concentration.

To prepare the tablet solutions, tablets containing $50 \mathrm{mg}$ or $100 \mathrm{mg}$ of PB were accurately weighed and crushed to a fine powder. A quantity equivalent of 10 $\mathrm{mg}$ PB was transferred into individual $100 \mathrm{~mL}$ volumetric flasks. After adding $70 \mathrm{~mL}$ of acetonitrile/water $(1: 1 ; \mathrm{v} / \mathrm{v})$, the flasks were vortex mixed for 3 minutes, followed by 10 minutes in ultrasonic bath. The samples were made up to volume with the same diluent. Working sample solutions were prepared prior to the analysis by diluting the stock solutions to the appropriate concentrations in mobilephase. Both sample and reference solutions were then filtered through a $0.45 \mu \mathrm{m}$ membrane filter (Millipore) prior to the injection.

\section{Apparatus and analytical conditions}

The LC method was performed on a Prominence HPLC system (Shimadzu, Kyoto, Japan) equipped with a CBM-20A system controller, an LC-20AD pump, a DGU-20A degasser, a SIL-20A autosampler and an
SPD-M20A photodiode array (PDA) detector. The peak areas were integrated automatically using an LCsolution software. The experiments were performed on a reversed phase Onyx ${ }^{\circledR} \mathrm{C} 18$ monolithic column (100 x 4.6 mm i.d., Phenomenex, Torrance, CA, USA). The LC system was operated isocratically at controlled temperature $\left(45^{\circ} \mathrm{C}\right)$ using as mobile phase acetonitrile and a solution of triethylamine $0.3 \% \mathrm{pH} 5.0$ (adjusted with phosphoric acid $8.5 \%)(50: 50 ; \mathrm{v} / \mathrm{v})$, run at a flow rate of $2.0 \mathrm{~mL} / \mathrm{min}$, and using PDA detection at 213 $\mathrm{nm}$. The injection volume was $10 \mu \mathrm{L}$.

\section{Validation procedure}

The objective of validation of an analytical procedure is to demonstrate that it is suitable for its intended purpose. The method was validated for specificity, linearity and range, precision, accuracy, detection limit, quantitation limit and robustness $(15,16)$.

\section{Specificity and forced degradation studies}

The specificity of an analytical method may be defined as the ability to determine the analyte in the presence of additional components such as impurities, degradation products, and matrix compounds (15). The interference of the excipients of the pharmaceutical formulation was determined by the injection of a sample containing only placebo (in-house mixture of all the tablet excipients) reference solution, and the commercial pharmaceutical preparation at the concentrations of $50 \mu \mathrm{g} / \mathrm{mL}$ of PB.

The stability-indicating capability of the method was determined by subjecting the reference solution (200 $\mu \mathrm{g} / \mathrm{mL})$ to accelerated degradation conditions such as: light exposure, acidic, basic and oxidative media to evaluate the interference in the analyte quantification. Hydrochloric acid was added to the sample solution to achieve the final concentration of $0.2 \mathrm{M}$ to verify the acid hydrolysis. The addition of sodium hydroxide to the sample solution to obtain a concentration of $1 \mathrm{M}$ was performed for the basic hydrolysis evaluation. Both solutions were neutralized with base and acid, respectively, after a pre-determined period of time. The oxidative degradation was induced by storing the sample solution in $15 \%$ hydrogen peroxide. All these solutions were kept in ambient temperature $\left(25^{\circ} \mathrm{C}\right)$ and protected from light to prevent the interference of photolytic degradation. Photodegradation was induced by exposing the sample in the photostability chambers to near UV-A $(365 \mathrm{~nm})$ and UV-C (254 nm) light for $4 \mathrm{~h}$ and $30 \mathrm{~min}$, respectively. After subjecting the samples to stress studies, they were withdrawn at suitable time intervals and diluted with the mobile phase to theoretical PB final concentrations of $50 \mu \mathrm{g} / \mathrm{mL}$ before LC injection. Then the specificity and the stability-indicating capability of the method were established by determining the PB peak purity using PDA detector.

Linearity and range 
The linearity was determined by constructing three independent analytical curves, each one with six concentrations of reference solution, in the range of 5$100 \mu \mathrm{g} / \mathrm{mL}(5 ; 10 ; 25 ; 50 ; 75 ; 100 \mu \mathrm{g} / \mathrm{mL})$ prepared in mobile-phase. Before injection of the solutions, the column was equilibrated for at least $20 \mathrm{~min}$ with the mobile phase flowing through the system. The peak areas of the compound were plotted against the respective concentration of $\mathrm{PB}$ to obtain the analytical curve.

\section{Precision and accuracy}

The precision of the method was determined by repeatability and intermediate precision. Repeatability was verified by six independent sample preparations of the same concentration of PB, on the same day, under the same experimental conditions. The intermediate precision was studied by comparing the results obtained on three different days. The accuracy was evaluated by applying the proposed method to the analysis of an in-house mixture of the excipients with known amounts of the drug, to obtain solutions at concentrations of 40,50 and $60 \mu \mathrm{g} / \mathrm{mL}$, equivalent to $80, \quad 100$ and $120 \%$ of the nominal analytical concentration, respectively. The accuracy was calculated as the percentage of the drug recovered from the formulation matrix.

\section{Detection/quantitation limits}

Detection/quantitation limits parameters are not a requirement for drug assay, however, it is always useful to demonstrate the sensitivity of the method and that the analysis is conducted in a region that is above of the quantitation limit value. The detection/quantitation limits were calculated from the analytical curve. Limits were calculated from the slope and the standard deviation of the intercept of three calibration curves, determined by a linear regression model, as defined by ICH guideline (15).

\section{Robustness}

In order to study the simultaneous variation of the factors in the considered responses, a multivariate approach using design of experiments (DoE) is recommended in robustness testing $(15,16)$. The DoE selected was a two-level fractional factorial design $\left(2^{4-}\right.$ $\left.{ }^{1}\right)$ performed to examine the effects of four factors (triethylamine solution $\mathrm{pH}$, triethylamine concentration, percentage of acetonitrile, and column temperature) at two levels in 9 experiments. The low and high levels were deliberately selected on the basis of small changes in method parameters, providing an indication of its reliability during normal usage. The obtained responses - drug assay (\%) and retention time (min) - were processed by Minitab 17 statistical software to evaluate the significance of the effects, which were represented by Pareto charts.
The stability of sample solutions in mobile phase was assessed after the storage of the samples for $48 \mathrm{~h}$ at 2-8 ${ }^{\circ} \mathrm{C}$, and also placed into the autosampler, at room temperature for $24 \mathrm{~h}$.

\section{Sample analysis}

For PB quantitation in the tablet formulations, the respective stock sample solutions were diluted to an appropriate concentration $(50 \mu \mathrm{g} / \mathrm{mL})$ with mobilephase, filtered, injected in triplicate and the percentage recoveries of the drug calculated against the reference substance.

\section{Results and Discussion}

\section{Method optimization}

The effect of the composition of the column and mobile-phase on the retention time of $\mathrm{PB}$ and on its chromatographic parameters was initially investigated. The chromatographic conditions were chosen after testing different mobile-phases with distinct proportions of organic solvent (acetonitrile or methanol) and water, with and without triethylamine, in different $\mathrm{pH}$ values of the aqueous phases. Phosphate buffers were also tested. The adequacy of the mobile-phase was decided on basis of selectivity and sensitivity of the assay, stability studies and the separation between the drug and degraded products formed during forced degradation studies. Moreover, the best chromatographic separation of degradation products and PB was achieved by using the monolithic column. The use of triethylamine solution $(0.3 \% ; \mathrm{pH}$ $5.0)$ in combination with acetonitrile $(50: 50 ; \mathrm{v} / \mathrm{v})$, at 45 ${ }^{\circ} \mathrm{C}$, resulted in a relatively short retention time of 3.4 min, and a simple mobile phase (without buffer addition), as shown in the typical chromatogram (Figure 2a). The optimized conditions of the LC method were validated for the analysis of $\mathrm{PB}$ in pharmaceuticals formulations, in order to provide capability and application for the quality control.

\section{Method Validation}

\section{Specificity and forced degradation studies}

A stability-indicating method is defined as an analytical method that accurately quantifies the active ingredients without interference from degradation products, process impurities, excipients, or other potential impurities (15). Forced degradation studies should be the first step in method development. The presence of degradants and impurities in pharmaceutical formulations can result in changes in their chemical, pharmacological and toxicological properties affecting their efficacy and safety. Therefore, the adoption of stability-indicating methods is always required to control the quality of pharmaceuticals during and after the production. This greatly contributes to the possibility of improving drug safety $(17,18)$.

\section{Solution stability}


During the forced degradations PB remained stable under basic and acidic hydrolysis, oxidative and UV-A photolytic conditions. However, the exposure of drug to UV-C (254 nm) light for $90 \mathrm{~min}$ resulted in full degradation of the drug. Exposing the PB for $20 \mathrm{~min}$, around $64 \%$ degradation was seen and five additional peaks were detected at $0.79,0.90,1.05,1.48$ and 2.32 min. Moreover, no interference from formulation excipients was found, showing that the drug peak were free from any co-eluting substance, thus demonstrating that the proposed method is specific for the analysis of PB. The chromatograms of forced degradation studies are shown in Figure 2.
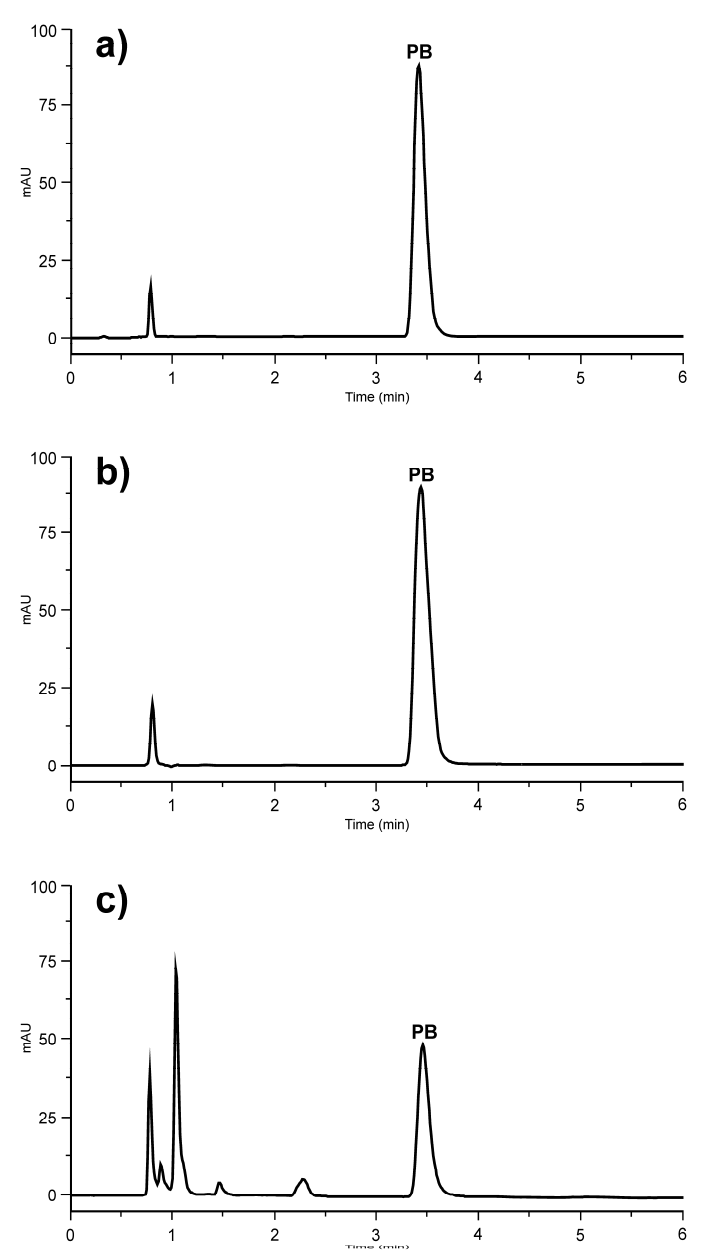

Figure 2. LC chromatograms of pinaverium bromide $(\mathrm{PB})$ at $50 \mu \mathrm{g} / \mathrm{mL}$. (a) PB reference substance solution; (b) PB tablet solution; (c) PB photolytic degradation in the photostability chambers to near UV-C $(254 \mathrm{~nm}) \quad(t=20 \mathrm{~min})$. Chromatographic conditions: Phenomenex Onyx $\mathrm{C} 18$ monolithic column (100 mm x $4.6 \mathrm{~mm}$ i.d.), $45{ }^{\circ} \mathrm{C}$; mobile phase: acetonitrile-triethylamine solution $0.3 \%$ (pH 5.0) (50:50; v/v); flow rate: $2.0 \mathrm{~mL} / \mathrm{min}$; injection: $10 \mu \mathrm{L}$.

\section{Linearity}

The analytical curves constructed for $\mathrm{PB}$ were found to be linear in the $5-100 \mu \mathrm{g} / \mathrm{mL}$ range. This range corresponded to $10-200 \%$ of the intended test concentration of $50 \mu \mathrm{g} / \mathrm{mL}$ for the pharmaceutical quality control of PB and the drug in its formulation.
The value of the determination coefficient calculated $\left(R^{2}=0.9991, y=3305 x-2098\right)$, where $x$ is concentration and $y$ is the peak absolute area, indicated the linearity of the analytical curve for the method.

\section{Precision}

Precision was determined by studying the intra-day and inter-day precision. The experimental values obtained for the determination of PB in samples are presented in Table 1. The variability of the results was low with RSD values less than $1.38 \%$ to intra-day precision, and the value of inter-day precision was $1.26 \%$ in tablets. RSD values found for the analytical method were within the acceptable range indicating that this method has excellent repeatability and intermediate precision.

Table 1. Intra-day and inter-day precision data of LC method for pinaverium bromide in tablet dosage form.

\begin{tabular}{|c|c|c|c|c|}
\hline Sample & Day 1 & Day 2 & & Day 3 \\
\hline 1 & $101.13 \%$ & $99.91 \%$ & & $98.59 \%$ \\
\hline 2 & $100.77 \%$ & $100.42 \%$ & & $100.60 \%$ \\
\hline 3 & $98.17 \%$ & $98.95 \%$ & & $102.23 \%$ \\
\hline 4 & $101.90 \%$ & $98.07 \%$ & & $100.29 \%$ \\
\hline 5 & $100.01 \%$ & $102.03 \%$ & & $99.62 \%$ \\
\hline 6 & $101.41 \%$ & $100.57 \%$ & & $99.57 \%$ \\
\hline $\begin{array}{l}\text { Intra-day } \\
\text { precision }^{\text {a }}(n=6)\end{array}$ & $\begin{array}{ll}100.57 \% \quad \pm \\
1.33 \%\end{array}$ & $\begin{array}{l}99.99 \% \\
1.38 \% \\
\end{array}$ & \pm & $\begin{array}{l}100.15 \% \\
\pm 1.23 \% \\
\end{array}$ \\
\hline $\begin{array}{l}\text { Inter-day } \\
\text { precision }^{\mathrm{a}}(n=18)\end{array}$ & \multicolumn{4}{|c|}{$100.24 \% \pm 1.26 \%$} \\
\hline
\end{tabular}

\section{Accuracy}

The accuracy was assessed from three replicate determinations of three different solutions containing 40, 50 and $60 \mu \mathrm{g} / \mathrm{mL}$. The absolute means obtained for PB are shown in Table 2 with a mean value of $100.68 \%$ and RSDs lower than $1.58 \%$, demonstrating that the method is accurate within the desired range.

Table 2. Accuracy data for proposed LC method.

\begin{tabular}{lccc}
\hline $\begin{array}{l}\text { Nominal } \\
\text { concentration } \\
(\boldsymbol{\mu g} / \mathbf{m L})\end{array}$ & $\begin{array}{c}\text { Mean } \\
\text { concentration } \\
\text { found }(\boldsymbol{\mu g} / \mathbf{m L})^{\mathbf{a}}\end{array}$ & $\begin{array}{l}\text { RSD } \\
(\boldsymbol{\%})^{\mathbf{b}}\end{array}$ & Accuracy $(\%)$ \\
\hline $40(80 \%)$ & 40.77 & 0.78 & 101.92 \\
$50(100 \%)$ & 50.19 & 0.45 & 100.37 \\
& & & \\
$60(120 \%)$ & 59.84 & 1.58 & 99.74 \\
\hline
\end{tabular}

${ }^{\text {a }}$ Mean of three replicates.

${ }^{\mathrm{b}} \mathrm{RSD}=$ Relative standard deviation. 


\section{Detection/quantitation limits}

Detection/quantitation limits were obtained by using the mean of the slope, $3302.86 \pm 140.54$ and the standard deviation of the intercept of the independent curves, determined by a linear regression line as 2495.13. The detection limit and quantitation limit calculated were 1.41 and $4.70 \mu \mathrm{g} / \mathrm{mL}$, respectively.

\section{Robustness}

The susceptibility of the developed analytical method to changes was tested in order to evaluate the robustness. For this purpose a two-level fractional factorial design was employed. The experimental plan and the corresponding responses are summarized in Table 3.

Table 3. Selected two-level fractional factorial design for the robustness testing of pinaverium bromide

\begin{tabular}{|c|c|c|c|c|c|c|}
\hline Experiment & $\begin{array}{c}\text { Factor } \\
\mathbf{A}^{\mathrm{a}}\end{array}$ & $\begin{array}{c}\text { Factor } \\
\mathbf{B}^{\mathbf{b}}\end{array}$ & $\begin{array}{c}\text { Factor } \\
\mathbf{C}^{\mathbf{c}}\end{array}$ & $\begin{array}{c}\text { Factor } \\
\mathbf{D}^{\mathbf{d}}\end{array}$ & $\begin{array}{c}\text { Assay } \\
(\%)\end{array}$ & $\begin{array}{c}\text { Retention } \\
\text { time (min) }\end{array}$ \\
\hline 1 & 4.8 & 52 & 0.2 & 47 & 101.17 & 3.19 \\
\hline 2 & 5.2 & 52 & 0.4 & 47 & 101.13 & 2.87 \\
\hline 3 & 4.8 & 52 & 0.4 & 43 & 100.86 & 2.79 \\
\hline 4 & 5.2 & 48 & 0.2 & 47 & 101.12 & 4.56 \\
\hline $5^{\mathrm{e}}$ & 5.0 & 50 & 0.3 & 45 & 101.07 & 3.43 \\
\hline 6 & 5.2 & 52 & 0.2 & 43 & 100.79 & 4.01 \\
\hline 7 & 5.2 & 48 & 0.4 & 43 & 101.10 & 3.48 \\
\hline 8 & 4.8 & 48 & 0.4 & 47 & 102.18 & 3.67 \\
\hline 9 & 4.8 & 48 & 0.2 & 43 & 101.49 & 4.46 \\
\hline \multicolumn{7}{|c|}{$\begin{array}{l}{ }^{\mathrm{a}} \text { Triethylamine } \mathrm{pH} \\
\mathrm{b} \text { Acetonitrile }(\%) \\
{ }^{\mathrm{c}} \text { Triethylamine concentration }(\%) \\
{ }^{\mathrm{d}} \text { Column temperature }\left({ }^{\circ} \mathrm{C}\right) \\
{ }^{\mathrm{e}} \text { Optimal conditions }\end{array}$} \\
\hline
\end{tabular}

The significance of the effects was evaluated by a Pareto chart (Figure 3) that consists of bars with a length proportional to the absolute value of the estimated effect, divided by the pseudo standard error defined by Lenth (Lenth's PSE) (19). The codes A, B, and $\mathrm{C}$ correspond to each of the parameters. The combination of two or three codes indicates the interaction effect between the variables. The bars were displayed based in the size of the effect, with the largest effect on the top. The chart includes a vertical line at the critical $t$-value $(\alpha=0.05)$. Effects in which the bars are smaller than the critical $t$-value were not considered significant and did not affect the response variables.
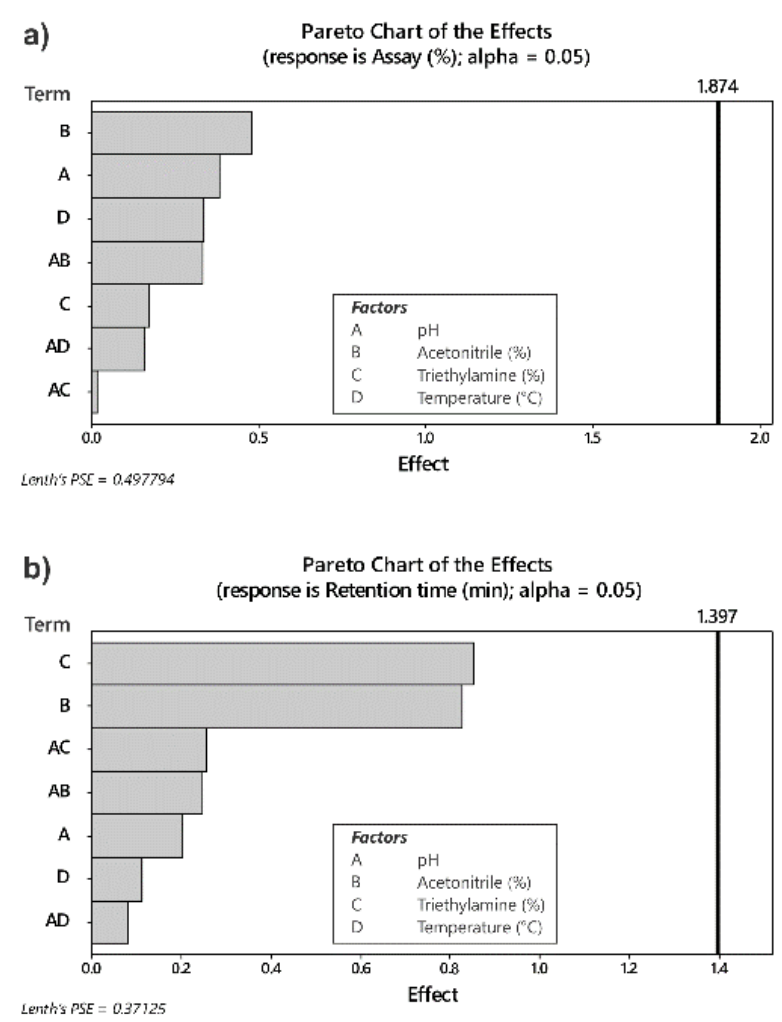

Figure 3. Pareto charts representing the effects of the variables and their interactions on the PB (a) assay and (b) retention time for the robustness test using the fractional factorial design.

\section{Solution stability}

The stability of the both reference and sample solutions was studied and the data obtained showed the stability during $24 \mathrm{~h}$ in the autosampler and during $48 \mathrm{~h}$ when maintained at $2-8{ }^{\circ} \mathrm{C}$. The retention time and peak area of PB remained almost unchanged and nonsignificant degradation was observed within the indicated period, which was sufficient for the whole analytical process.

\section{Method Application}

Acceptable results of application of the proposed method in tablet dosage forms (Table 2) were obtained, demonstrating the quality of the pharmaceutical samples and the applicability of the validated method for quality control analysis of PB. It should be considered that excipients did not interfere in the determination of the active compound.

\section{Conclusions}

The high-throughput assay is an extremely important advantage of LC methods using monolithic columns, especially during new product development and validation of manufacturing processes. The results of the validation studies showed that the stability indicating LC method is specific, accurate, robust, and 
possesses significant linearity and precision, without any interference from the excipients and degradation products, according to ICH requirements. The method is fast, selective, and requires a simple sample preparation procedure. Thus, the proposed LC method was successfully applied for the quantitative analysis of $\mathrm{PB}$ in tablet dosage forms and can be employed for drug analysis during stability studies, contributing to the improvement of quality control.

\section{Acknowledgements}

The authors wish to thank FAPERJ and PIBICUFRJ for the financial support.

\section{References}

1. Ikechi, R., Fischer, B.D., DeSipio, J., Phadtare, S. Irritable bowel syndrome: clinical manifestations, dietary influences, and management. Healthcare. 2017; 5(21): 1-14.

2. World Gastroenterology Organisation (WGO). Global Guidelines Irritable Bowel Syndrome: a Global Perspective. Milwaukee, USA, 2015. $28 \mathrm{p}$.

3. Weaver, K.R., Melkus, G.D., Henderson, W.A. Irritable bowel syndrome. Am J Nurs. 2017; 117(6): 48-55.

4. Annaházi, A., Róka, R., Rosztóczy, A., Wittmann, T. Role of antispasmodics in the treatment of irritable bowel syndrome. World J Gastroenterol. 2014; 20(20): 6031-6043.

5. Zheng, L., Lai, Y., Lu, W., Li, B., Fan, H., Yan, $Z$., et al. Pinaverium reduces symptoms of irritable bowel syndrome in a multicenter, randomized, controlled trial. Clin Gastroenterol H. 2015; 13: 1285-1292.

6. Ramachandra, B. Development of impurity profiling methods using modern analytical techniques. Crit Rev Anal Chem. 2017; 47(1): 24-36.

7. Kątny, M., Frankowski, M.. Impurities in drug products and active pharmaceutical ingredients. Crit Rev Anal Chem. 2017; 47(3): 187-193.

8. Sahu, P.K., Ramisetti, N.R., Cecchi, T., Swain, S., Patro, C.S., Panda, J. An overview of experimental designs in HPLC method development and validation. J Pharm Biomed Anal. 2017; in press.

9. Skibinski, R., Trawinski, J. Application of an untargeted chemometric strategy in the impurity profiling of pharmaceuticals: an example of amisulpride. J Chromatogr Sci. 2017; 55(3): 309-315.
10. Holm, R., Elder, D.P. Analytical advances in pharmaceutical impurity profiling. Eur J Pharm Sci. 2016; 87: 118-135.

11. Jandera, P., Hájek, T., Sandra, P. Monolithic and core-shell columns in comprehensive twodimensional HPLC: a review. Anal Bioanal Chem. 2015; 407: 139-151.

12. Sangoi, M.S., Todeschini, V., Steppe, M.. Monolithic LC method applied to fesoterodine fumarate low dose extended-release tablets: dissolution and release kinetics. J Pharm Anal. 2015; 5(2): 137-141.

13. Ibrahim, F., Wahba, M.E.K. Simultaneous liquid chromatographic determination of ebastine with two sympathomimetic drugs using a monolithic column. J Chromatogr Sci. 2017; 55(3): 258-266.

14. Martins, D.F.C., Florindo, L.C., Machado, A.K.M.S., Todeschini, V., Sangoi, M.S. Bromide pinaverium: development and validation of spectrophotometric methods for assay and dissolution studies. J AOAC Int. 2017; in press.

15. International Conference on Harmonization (ICH) of Technical Requirements for the Registration of Pharmaceutical for Human Use. Validation of Analytical Procedures: Text and Methodology Q2(R1). Geneva, Switzerland, 2005. 17p.

16. Food and Drug Administration (FDA), Center for Drug Evaluation and Research. Guidance for Industry: Analytical Procedures and Methods Validation for Drugs and Biologics. Silver Spring, USA, 2015. 18p.

17. Singh, S., Junwal, M., Modhe, G., Tiwari, H., Kurmi, M., Parashar, N., et al. Forced degradation studies to assess the stability of drugs and products. Trac-Trend Anal Chem. 2013; 49: 71-88.

18. Blessy, M., Patel, R.D., Prajapati, P.N., Agrawal, Y.K. Development of forced degradation and stability indicating studies of drugs - a review. J Pharm Analysis. 2014; 4(3): 159-165.

19. Lenth, R.V. Quick and easy analysis of unreplicated factorials. Technometrics. 1989; 31: 469-473. 